\title{
INVESTIGAÇÃO DO MECANISMO DE CATÁLISE ROMP DO NORBORNENO UTILIZANDO MÉTODOS DE FUNCIONAL DE DENSIDADE
}

\author{
Carlos Pereira da Silva* \\ Instituto Federal de Educação, Ciência e Tecnologia do Piauí, Campus Prof. Marcílio Rangel Farias, 64018-000 Teresina - PI, Brasil \\ Francisco das Chagas Alves Lima \\ Coordenação de Química, Universidade Estadual do Piauí, 64002-150 Teresina - PI, Brasil \\ Régis Casimiro Leal e José Machado Moita Neto \\ Departamento de Química, Centro de Ciências da Natureza, Universidade Federal do Piauí, 64049-550 Teresina - PI, Brasil
}

Recebido em 23/7/09; aceito em 14/3/10; publicado na web em 9/8/10

\begin{abstract}
INVESTIGATION OF THE ROMP CATALYSIS MECHANISM OF NORBORNENE USING METHODS OF DENSITY FUNCTIONAL. This work presents a density functional theory study of the norbornene ROMP metathesis reactions. The energies have been calculated in a Grubbs catalyst model $\mathrm{Cl}_{2}\left(\mathrm{PH}_{3}\right)_{2} \mathrm{Ru}=\mathrm{CH}_{2}$. The geometries and energy profile are similar to the Grubbs metilydene $\left(\mathrm{Cl}_{2}\left(\mathrm{PCy}_{3}\right)_{2} \mathrm{Ru}=\mathrm{CH}_{2}\right.$ real model. It was found that the metathesis reaction proceeds via associative mechanism (catalyst-norbonene) followed by dissociative substitution of a phosphine ligand with norbonene, giving a monophosphine complex. The results are in reasonable agreement with the available experimental data. The dissociation energy of the phosphines is predicted to be $23.2 \mathrm{kcal} \mathrm{mol}^{-1}$.
\end{abstract}

Keywords: ROMP-metathesis; molecular modeling; DFT.

\section{INTRODUÇÃO}

Derivada das palavras gregas meta - mudança e thesis - posição, a metátese é a troca de partes de duas substâncias. ${ }^{1}$ Quando ocorre tal troca de grupos entre duas olefinas iniciais, duas outras olefinas são produzidas. Catalisadores para metátese foram desenvolvidos como ferramentas altamente poderosas e versáteis em síntese orgânica. A grande variedade de transformações sintéticas que podem ser realizadas é surpreendente. Somente com Y. Chauvin, em 1971, que um mecanismo viável foi apresentado. Chauvin e Hérisson propuseram que a reação se iniciava com a formação de um complexo metalcarbeno $^{1,2}$ e publicaram o mecanismo da metátese como ilustrado na Figura 1.

Inicialmente, ocorre uma adição do tipo $2+2$ do complexo metalcarbeno com a olefina. Logo em seguida acontece a coordenação da olefina com o complexo metal-carbeno, formando um intermediário metalociclobutano. Por fim, uma cicloreversão dá origem a um novo complexo metal-carbeno. Dentre os diversos tipos de metátese destacam-se: ${ }^{1} \mathrm{CM}$ (cross-metathesis), metátese cruzada - ocorre entre olefinas diferentes; RCM (ring-closing metathesis), metátese de fechamento de anel - é uma metátese intramolecular que ocorre em dienos, na qual há formação de um anel; ROM (ring-opening metathesis), metátese com abertura de anel - ocorre abertura de uma ciclo-olefina formando dienos, é o inverso da RCM; ADMET (acyclic diene metathesis polymerization), polimerização via metátese entre dienos acíclicos; e ROMP (ring-opening metathesis polymerization), polimerização via metátese com abertura de anel - ocorre entre alcenos cíclicos, único método de polimerização de inúmeros polímeros que não podem ser obtidos por outros processos. ${ }^{3}$ Grubbs e colaboradores, em 1992, publicaram o primeiro complexo molecular metal-carbeno bem definido $\mathrm{Cl}_{2}\left(\mathrm{PPh}_{3}\right)_{2} \mathrm{Ru}=\mathrm{CH}-\mathrm{CH}=\mathrm{CPh}_{2}$, que não somente era ativo para a polimerização do norborneno como também era estável na presença de solventes próticos. ${ }^{4}$ Para aumentar a reatividade do catalisador, os grupos fenil foram trocados por grupos ciclo-hexil $(\mathrm{R}=\mathrm{Cy})$. Em 1995, Grubbs publicou novos catalisado-

*e-mail: carlosps1985@gmail.com

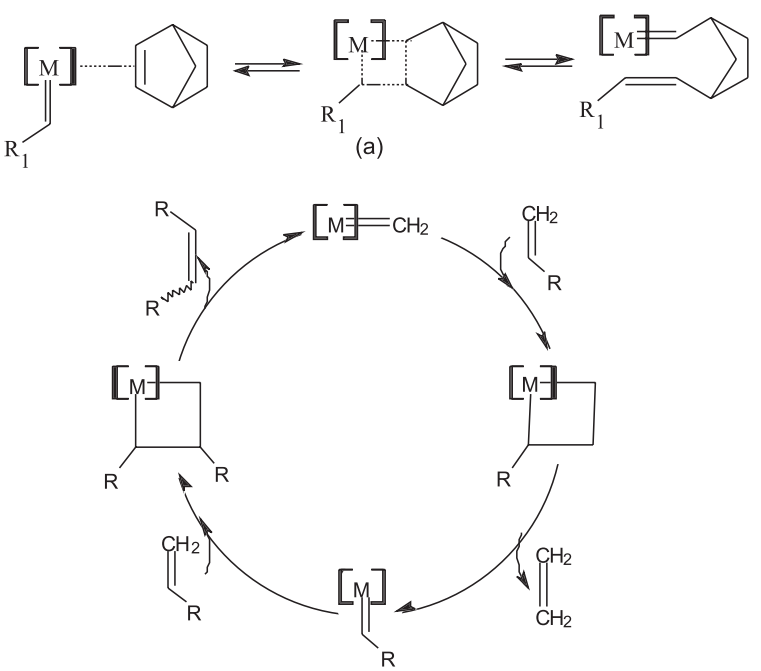

(b)

Figura 1. Ciclo catalítico para metátese com complexos metal-carbeno: (a) metátese do norborneno, (b) esquema geral

res molecularmente bem definidos $\mathrm{Cl}_{2}\left(\mathrm{PR}_{3}\right)_{2} \mathrm{Ru}=\mathrm{CHPh}, \mathrm{R}=\mathrm{Ph}$ ou $\mathrm{Cy}$ (ciclo-hexil). ${ }^{5} \mathrm{~A}$ combinação ficou conhecida como a primeira geração dos catalisadores de Grubbs. Atualmente, $\mathrm{Cl}_{2}[\mathrm{C}(\mathrm{N}$ (mesityl) $\left.\left.\mathrm{CH}_{2}\right)_{2}\right]\left(\mathrm{PCy}_{3}\right) \mathrm{Ru}=\mathrm{CHPh}$ é o catalisador mais utilizado para realizar determinados tipos de metátese. Este novo catalisador de rutênio, com sua maior estabilidade térmica faz parte da segunda geração dos catalisadores de Grubbs.

Entender como as reações ocorrem em nível atômico, saber seus detalhes, os motivos de determinadas reações ocorrerem e outras não, umas serem rápidas e outras lentas, os fatores responsáveis pela proporção de cada produto, é muito importante no controle e design de novos catalisadores mais eficientes. Algumas teorias foram propostas a fim de auxiliar no entendimento deste processo químico. Experimentalmente é uma tarefa difícil, o que se deve às pequenas distâncias interatômicas e às curtas escalas de tempo envolvidas. 
Trabalhos com espectroscopia de femtossegundos $\left(10^{-15} \mathrm{~s}\right)$ começaram a responder alguns destes questionamentos, ${ }^{6} \mathrm{e}$ os resultados computacionais de estruturas de transição também contribuem para o estabelecimento dos mecanismos. O foco principal deste estudo foi a análise do mecanismo de trimerização do norborneno como uma simulação simplificada da sua polimerização, via metátese ROMP, utilizando cálculos DFT. Vários caminhos de reações são possíveis e os de menor energia são favorecidos. O mecanismo mais comum e que ocorre em maior proporção (em torno de $95 \%$ das reações) é o chamado dissociativo, ${ }^{7}$ no qual, antes de haver formação de intermediários, ocorre dissociação de uma fosfina. Essa dissociação pode ocorrer antes ou depois da primeira interação catalisador-olefina, porém a literatura experimental não distingue estes dois caminhos. ${ }^{7}$

\section{PARTE EXPERIMENTAL}

O custo computacional para o cálculo de integrais de 1 e 2 elétrons em moléculas com muitos átomos e com metal, como os catalisadores de Grubbs, é muito grande, demandando muito tempo e capacidade computacional. Sendo assim, o ciclo catalítico completo para o sistema em estudo foi feito com o análogo simplificado $\mathrm{Cl}_{2}\left(\mathrm{PH}_{3}\right)_{2} \mathrm{Ru}=\mathrm{CH}_{2}$. Os cálculos computacionais foram executados através de métodos de Química Quântica baseados na Teoria do Funcional da Densidade (DFT), utilizando o pacote computacional Gaussian 03W. ${ }^{8} \mathrm{O}$ funcional utilizado foi o B3LYP, ${ }^{9}$ que tem se mostrado bastante útil para descrever sistemas contendo metais de transição, ${ }^{10}$ com o conjunto de base lanl2dz. Todas as estruturas consideradas pontos estacionários foram submetidas à análise de frequências, para serem classificadas como geometria de equilíbrio ou estados de transição. Os cálculos foram realizados considerando-se os elétrons das estruturas metalocarbenos formando singleto, por esta forma ser a mais estável ${ }^{11}$ e considerando a carga total como sendo nula, como previsto em trabalhos anteriores. ${ }^{12}$ Para obtenção dos estados de transição, inicialmente, foram otimizadas as geometrias dos reagentes e produtos correspondentes a cada um com o funcional B3LYP e conjunto de base lanl2dz. A obtenção dos estados de transição foi realizada utilizando-se o método Synchronous TransitGuided Quasi-Newton (STQN), com as opções QST2 e QST3. A opção QST2 requer a especificação das estruturas do reagente e do produto; quando o método QST2 não funciona a saída do cálculo sugere a busca com o método QST3. Além das estruturas do reagente e do produto, no método QST3, é necessário incluir uma estrutura inicial para o estado de transição. Como resultado é gerada uma estrutura de transição, que é um intermediário entre os reagentes e produtos, em termos de coordenadas internas redundantes. Estes estados de transição foram, em seguida, otimizados e submetidos a cálculos de constantes de força; ao se obter uma constante de força negativa única pode-se concluir que se trata de um estado de transição legítimo. Foram feitos cálculos IRC (Intrinsic Reaction Coordinate), para verificar se na coordenada intrínseca de reação as energias eram descendentes nos sentidos "Estado de Transição $\rightarrow$ Reagentes" e "Estado de Transição $\rightarrow$ Produtos".

\section{RESULTADOS E DISCUSSÃO}

\section{Estrutura do catalisador}

A Tabela 1 mostra uma comparação entre os parâmetros estruturais obtidos por cálculos B3LYP/lanl2dz para o catalisador $\mathrm{Cl}_{2}\left(\mathrm{PH}_{3}\right)_{2} \mathrm{Ru}=\mathrm{CH}_{2}$ e dados de difração de raios $\mathrm{X}$ de análogos reais, $\mathrm{r}_{\mathrm{x}}=\mathrm{Cl}_{2}(\mathrm{PCy})_{2} \mathrm{Ru}=\mathrm{CHR}$, com R=-orto- $\mathrm{Ph}-\mathrm{Cl}\left(\mathrm{r}_{1}\right)^{5},-\mathrm{O}-\mathrm{CH}=\mathrm{CH}_{2}\left(\mathrm{r}_{2}\right)^{13}$ e $-\mathrm{CH}=\mathrm{C}\left(\mathrm{Ph}_{2}\right)\left(\mathrm{r}_{3}\right) \cdot{ }^{14}$ Todas as estruturas possuem uma geometria piramidal distorcida em torno do centro metálico; os cálculos mostram a ligação $\mathrm{Ru}=\mathrm{C}$ com um ângulo de $91,9^{\circ}$ em relação a P2 (Figura 2), perpendicular ao eixo $\mathrm{P} 1-\mathrm{P} 2$, e Cl1-Ru-Cl2 com 147, $0^{\circ}$. Este valor maior que $120^{\circ}$ mostra que o sistema não adota uma estrutura bipiramidal. As fosfinas apresentam-se em posição trans.

Tabela 1. Comprimentos de ligação (angstroms/Å) e ângulos (graus/ ${ }^{\circ}$ ) calculados para o catalisador e valores obtidos experimentalmente por difração de raios $\mathrm{X}$ para os complexos $\mathrm{r}_{1}, \mathrm{r}_{2}$ e $\mathrm{r}_{3}$

\begin{tabular}{lcccc}
\hline $\begin{array}{l}\text { Parâmetros } \\
\text { geométricos }\end{array}$ & $\begin{array}{c}\text { DFT (B3LYP/ } \\
\text { lan12dz) }\end{array}$ & $\mathrm{r}_{1}$ & $\begin{array}{c}\text { Raios X } \\
\mathrm{r}_{2}\end{array}$ & $\mathrm{r}_{3}$ \\
\hline $\mathrm{r}(\mathrm{Ru}=\mathrm{C})$ & 1,840 & 1,839 & 1,812 & 1,851 \\
$\mathrm{r}(\mathrm{Ru}-\mathrm{X} 1)$ & 2,423 & 2,401 & 2,388 & 2,401 \\
$\mathrm{r}(\mathrm{Ru}-\mathrm{X} 2)$ & 2,423 & 2,395 & 2,374 & 2,391 \\
$\mathrm{r}(\mathrm{Ru}-\mathrm{L} 1)$ & 2,436 & 2,397 & 2,368 & 2,413 \\
$\mathrm{r}(\mathrm{Ru}-\mathrm{L} 2)$ & 2,436 & 2,435 & 2,390 & 2,423 \\
$\angle(\mathrm{C}=\mathrm{Ru}-\mathrm{L} 1)$ & 91,9 & 97,5 & 97,2 & 98,7 \\
$\angle(\mathrm{C}=\mathrm{Ru}-\mathrm{L} 2)$ & 91,9 & 101,2 & 98,0 & 98,9 \\
$\angle(\mathrm{C}=\mathrm{Ru}-\mathrm{X} 1)$ & 106,5 & 88,7 & 97,3 & 88,8 \\
$\angle(\mathrm{C}=\mathrm{Ru}-\mathrm{X} 2)$ & 106,5 & 103,7 & 89,1 & 97,8 \\
$\angle(\mathrm{X} 1-\mathrm{Ru}-\mathrm{X} 2)$ & 146,9 & 167,6 & 173,5 & 173,4 \\
$\angle(\mathrm{L} 1-\mathrm{Ru}-\mathrm{L} 2)$ & 176,1 & 161,1 & 164,6 & 162,2 \\
$\angle(\mathrm{X} 1-\mathrm{Ru}-\mathrm{L} 1)$ & 90,0 & 87,2 & 91,6 & 89,2 \\
$\angle(\mathrm{X} 1-\mathrm{Ru}-\mathrm{L} 2)$ & 88,8 & 90,8 & 88,1 & 88,3 \\
$\angle(\mathrm{X} 2-\mathrm{Ru}-\mathrm{L} 1)$ & 88,8 & 91,5 & 87,5 & 89,5 \\
$\angle(\mathrm{X} 2-\mathrm{Ru}-\mathrm{L} 2)$ & 90,0 & 86,5 & 90,8 & 91,0 \\
\hline
\end{tabular}

Para avaliar se o modelo adotado é semelhante a catalisadores reais, foi utilizado o teste $\mathrm{t}$ pareado em uma comparação entre cálculos e dados de raios X para comprimentos de ligação e ângulos. Os resultados são mostrados na Tabela 1. Os valores obtidos de $\mathrm{p}$ foram 0,95 (catalisador e $r_{1}$ ), 0,99 (catalisador e $r_{2}$ ), 0,99 (catalisador e $r_{3}$ ). Portanto, os resultados calculados reproduzem bem os valores obtidos experimentalmente, dentro do intervalo de confiança de $95 \%$. Como a semelhança estrutural entre catalisadores de Grubbs de primeira geração e os análogos $r_{x}$ é muito grande, os parâmetros destes, que são semelhantes ao modelo adotado, podem ser utilizados com razoável aproximação como referência. As estruturas mantêm a mesma simetria e os parâmetros geométricos são bem semelhantes. Em geral, as distâncias de ligações em torno do centro metálico obtidas nos cálculos são maiores que as observadas experimentalmente e, com relação aos ângulos, verifica-se um distanciamento entre os haletos e a ligação $\pi$.

\section{Mecanismo geral para metátese ROMP do norborneno}

Modelos experimentais e teóricos ${ }^{7,15,16}$ permitiram descrever um mecanismo para trimerização do norborneno; a reação modelo é finalizada com adição de uma molécula de eteno. As estruturas presentes no mecanismo em questão são mostradas na Figura 2; o mecanismo proposto pode ser visto por completo na Figura $1 \mathrm{~S}$, material suplementar.

\section{Iniciação - dissociação da fosfina}

Quando ocorre a metátese de uma olefina pelo mecanismo dissociativo se forma uma nova olefina, que pode possuir isomeria geométrica. Podem ser originadas olefinas cis e trans, isso dependerá do alceno em questão e da forma como este se liga ao complexo. Caso o mecanismo seja o associativo só serão produzidas olefinas cis. Este processo está ilustrado na Figura 3.

Estudos do mecanismo de reações de metátese de olefinas com catalisadores de rutênio mostram que para complexos de rutênio 


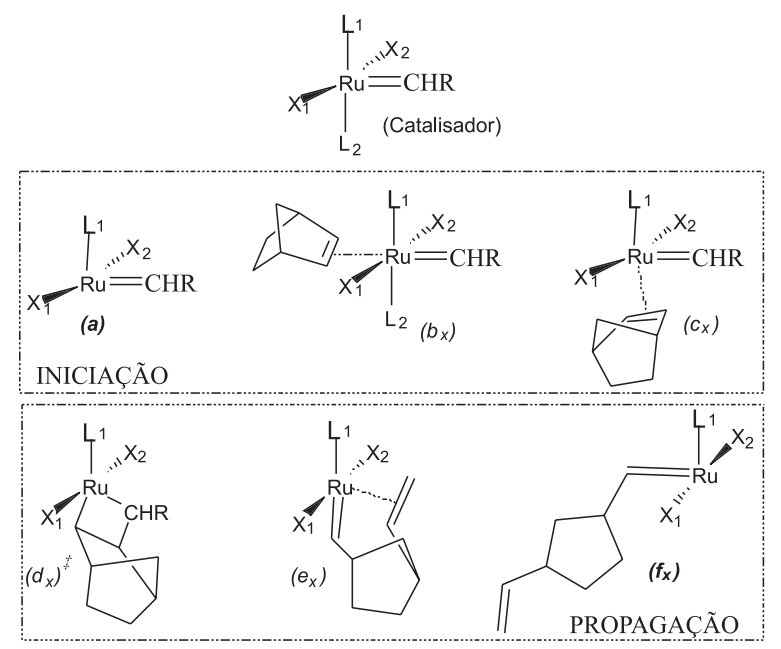

Figura 2. Estruturas presentes no mecanismo dissociativo em estudo, com: $L=P H_{3}, X=C l$ e $R=H$. $O$ índice " $x$ " refere-se ao número de moléculas de norborneno já presentes na estrutura, no desenho acima $x=1$ (para trimerização $x$ vai 1 a 3 )

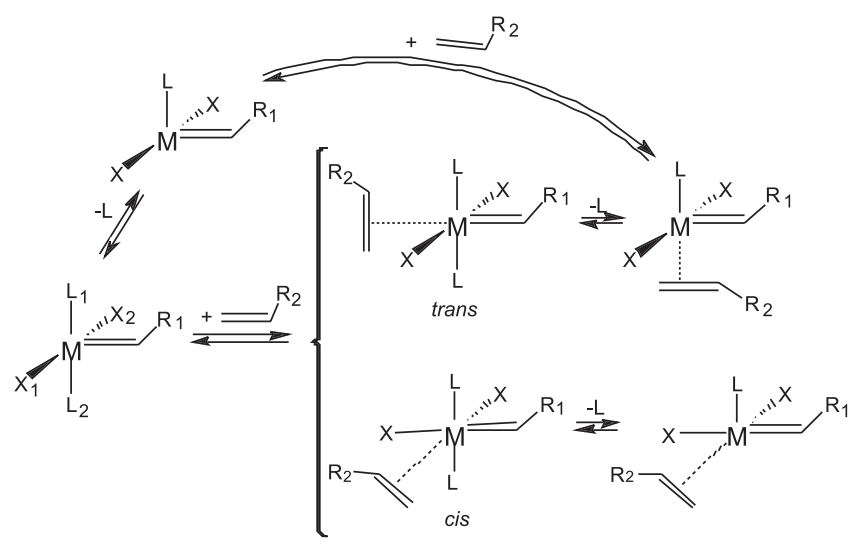

Figura 3. Possíveis mecanismos de iniciação em uma reação de metátese

com a fórmula geral $\mathrm{X}_{2}(\mathrm{~L})\left(\mathrm{PR}_{3}\right) \mathrm{Ru}=\mathrm{CHR}(\mathrm{R}=\mathrm{Cy}, \mathrm{Cp}$ e $\mathrm{Ph} ; \mathrm{X}=\mathrm{Cl}$, Br e I; L=ligante N-heterocíclico) a iniciação ocorre pelo mecanismo dissociativo, no qual uma fosfina é substituída por um substrato olefínico. ${ }^{13,14,16}$

Para iniciar o processo de catálise a primeira etapa, neste caso a dissociação de uma fosfina, deve ter uma energia adequada. Para confirmar a orientação da ligação dupla olefínica em relação ao ligante $\mathrm{PH}_{3}$ e verificar qual mecanismo é termodinamicamente mais favorável para o modelo de metátese em estudo, foi realizado um cálculo das energias envolvidas na etapa inicial do processo. As energias relativas encontradas, considerando $\mathrm{E}_{\text {catalisador }}=0 \mathrm{kcal} \mathrm{mol}^{-}$ 1, foram: $\mathrm{E}_{a}=23,2 ; \mathrm{E}_{b l}=-1,4 \mathrm{e} \mathrm{E}_{c l}=16,3 \mathrm{kcal} \mathrm{mol}^{-1}$ (Figuras $2 \mathrm{e}$ 4). A entalpia observada para a etapa de troca fosfínica (catalisador $\rightarrow c_{l}$ ) foi de $16,0 \mathrm{kcal} \mathrm{mol}^{-1}$, Grubbs publicou um trabalho ${ }^{7}$ onde encontrou uma entalpia de 19,0 kcal mol-1, para um catalisador com $\mathrm{R}=\mathrm{Ph}, \mathrm{X}=\mathrm{I}$ e $\mathrm{L}=\mathrm{PCy}_{3}$. A natureza dos grupos ligados ao centro metálico influencia a catálise, ${ }^{11,17,18}$ e os efeitos podem variar de acordo com a etapa em questão (iniciação ou propagação). Ligantes mais volumosos, em geral, favorecem a propagação de reações de metátese,,$^{18}$ apesar de dificultar a dissociação da fosfina. As energias eletrônicas de dissociação do ligante $(\Delta \mathrm{E})$ foram calculadas para o grupo $\mathrm{PH}_{3}$. A energia de dissociação de uma fosfina do catalisador $\mathrm{Cl}_{2}\left(\mathrm{PH}_{3}\right)_{2} \mathrm{Ru}=\mathrm{CH}_{2}$ com formação de um complexo $\pi$ entre o metal e a olefina, é representada pela energia relativa da estrutura $a(23,2$ $\left.\mathrm{kcal} \mathrm{mol}^{-1}\right)$ e a variação total de energia $(\Delta \mathrm{E})$ do processo é termodinamicamente favorável com o valor de $-41,4 \mathrm{kcal} \mathrm{mol}^{-1}(\Delta \mathrm{H}=-36,0$ $\left.\mathrm{kcal} \mathrm{mol}^{-1}\right)$. A dissociação precedida da interação catalisador-olefina libera $1,4 \mathrm{kcal} \mathrm{mol}^{-1}$ de energia $\left(\Delta \mathrm{H}=-1,4 \mathrm{kcal} \mathrm{mol}^{-1}\right)$. Uma dissociação sem prévia interação catalisador-olefina aumenta a energia do sistema $\left(\mathrm{E}_{a}\right)$, a variação de energia que ocorre entre as estruturas $b_{1}$ e $c_{1}$ é de $17,7 \mathrm{kcal} \mathrm{mol}^{-1}$. Portanto, a dissociação ocorre somente após uma interação catalisador-olefina, como sugerido por Grubbs em estudos cinéticos. ${ }^{19}$

Ligantes mais volumosos, como $\mathrm{PCy}_{3}$, favorecem a dissociação da fosfina oposta $\left(\mathrm{PH}_{3}\right)$, estabilizando as estruturas $a$ e $c_{1}$. Apesar do efeito estérico deste grupo dificultar a entrada de uma nova olefina, trabalhos experimentais ${ }^{7,19}$ mostram que a dissociação do grupo $\mathrm{PH}_{3}$ não é favorecida pelo aumento do efeito estérico, mas sim pela doação de densidade eletrônica do ligante para o metal. Para analisar o efeito de um ligante mais básico na iniciação da metátese foram feitos cálculos para um análogo do tipo $\mathrm{Cl}_{2}\left(\mathrm{PCy}_{3}\right)\left(\mathrm{PH}_{3}\right) \mathrm{Ru}=\mathrm{CH}_{2}$, as energias obtidas, considerando $\mathrm{E}\left(\mathrm{Cl}_{2}\left(\mathrm{PCy}_{3}\right)\left(\mathrm{PH}_{3}\right) \mathrm{Ru}=\mathrm{CH}_{2}\right)=0 \mathrm{kcal}$ mol ${ }^{-1}$, foram: $\mathrm{E}_{a}=16,6 ; \mathrm{E}_{b l}=-4,4 \mathrm{e} \mathrm{E}_{c l}=7,0 \mathrm{kcal} \mathrm{mol}^{-1}$, e os resultados são mostrados na Figura 4. Uma comparação entre os valores de energia envolvidos na dissociação dos catalisadores $\mathrm{Cl}_{2}\left(\mathrm{PH}_{3}\right)_{2} \mathrm{Ru}=\mathrm{CH}_{2}$ $\mathrm{e} \mathrm{Cl}_{2}\left(\mathrm{PCy}_{3}\right)\left(\mathrm{PH}_{3}\right) \mathrm{Ru}=\mathrm{CH}_{2}$ mostra que uma fosfina mais básica, como $\mathrm{PCy}_{3}$, gera uma estabilização das estruturas e as energias observadas são menores. Quanto à diminuição de energia devido à interação catalisador-norborneno os valores observados são menores tanto para o mecanismo dissociativo ( $a^{\prime} \rightarrow c_{1}$ ' / Figura 4$), \Delta \mathrm{E}=-9,6 \mathrm{kcal} \mathrm{mol}^{-1}$, quanto para o mecanismo associativo (catalisador' $\rightarrow b_{1}$ ' / Figura 4), $\Delta \mathrm{E}=-4,4 \mathrm{kcal} \mathrm{mol}^{-1}$.

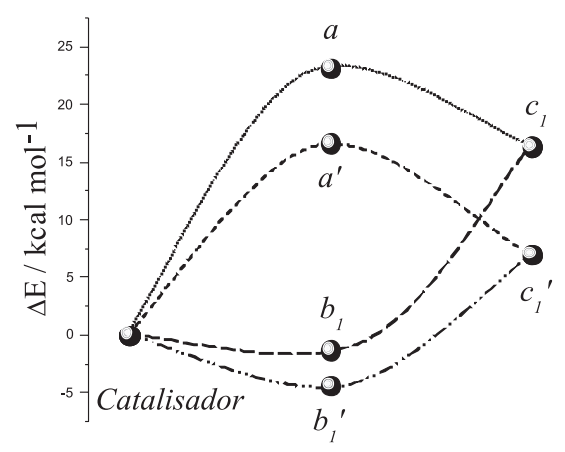

Figura 4. Perfis de energia relativa* (B3LYP/lanl2dz) envolvidos na dissociação da fosfina. Os cálculos foram realizados para as estruturas $\mathrm{Cl}_{2}\left(\mathrm{PH}_{3}\right)_{2} \mathrm{Ru}=\mathrm{CH}_{2}$ (Catalisador - dissociativo, associativo) e $\mathrm{Cl}_{2}\left(\mathrm{PCy}_{3}\right)$ $\left(\mathrm{PH}_{3}\right) \mathrm{Ru}=\mathrm{CH}_{2}$ (Catalisador'- dissociativo, associativo). *As energias dos catalisadores foram consideradas como sendo zero

\section{Cargas e superfícies de potencial eletrostático (SPE) durante a dissociação}

Para investigar o comportamento e influência da densidade eletrônica no processo de dissociação foram feitos cálculos de potencial eletrostático, cargas atômicas e orbitais de fronteira. Os resultados podem ser vistos nas Figuras $2 \mathrm{~S}, 3 \mathrm{~S}, 4 \mathrm{~S}$ e $5 \mathrm{~S}$, material suplementar, e Tabela 2. Uma análise qualitativa das SPE's sugere que uma prévia dissociação do catalisador aumenta a concentração das cargas atômicas. O maior momento de dipolo encontrado dentre as cinco estruturas (Figura 2S) foi de 5,6 D para estrutura $a$, seguido da estrutura $c_{1} \operatorname{com} 2,9 \mathrm{D}$. As cargas atômicas na estrutura $a$ são as que mais diferem das demais; é a única estrutura onde o rutênio apresenta carga positiva. Uma análise detalhada permite verificar que uma dissociação sem prévia interação catalisadorolefina (catalisador $\rightarrow a \rightarrow c_{1}$ / Figura 1S) aumenta as chances de 
haver competição entre a entrada da olefina na estrutura $a$ e a volta da fosfina, o que diminuiria o rendimento da reação. Estes fatores sugerem que a dissociação deve ocorrer após a interação.

Tabela 2. Cargas de Mulliken ( $e^{-}$) calculadas (B3LYP/lanl2dz) para espécies envolvidas na dissociação da fosfina

\begin{tabular}{lcccc}
\hline Átomos & \multicolumn{5}{c}{ Cargas } \\
\hline $\mathrm{Ru}$ & Catalisador & $\mathrm{a}^{\mathrm{c}}$ & $\mathrm{b}_{1}$ & $\mathrm{c}_{1}$ \\
$\mathrm{C}$ & $-0,02$ & 0,08 & $-0,04$ & $-0,08$ \\
$\mathrm{C} 11$ & $-0,44$ & $-0,44$ & $-0,44$ & $-0,45$ \\
$\mathrm{Cl} 2$ & $-0,26$ & $-0,25$ & $-0,26$ & $-0,26$ \\
$\mathrm{P} 1$ & $-0,26$ & $-0,25$ & $-0,26$ & $-0,26$ \\
$\mathrm{P} 2$ & 0,12 & 0,20 & 0,12 & 0,19 \\
\hline
\end{tabular}

Os orbitais de fronteira do complexo $\pi$ (catalisador-olefina) são formados por uma contribuição significativa do HOMO do norborneno e LUMO do catalisador. Este fato pode ser observado de forma qualitativa na Figura 3S, material suplementar, onde são mostrados os orbitais de fronteira das estruturas iniciais e do complexo $\pi$.

Se considerarmos os eixos x e y, como sendo representados pelas ligações $\mathrm{Ru}=\mathrm{C}$ e $\mathrm{Ru}-\mathrm{P}$, respectivamente, o LUMO da estrutura $a$ $\left(\mathrm{Cl}_{2}\left(\mathrm{PH}_{3}\right) \mathrm{Ru}=\mathrm{CH}_{2}\right)$ é predominantemente o orbital $\mathrm{dx}^{2}-\mathrm{y}^{2}$ do rutênio com um lóbulo no sítio de coordenação vazio (eixo y), o HOMO corresponde basicamente ao orbital dyz e o HOMO-1 ao dxz. Estes orbitais estão idealmente orientados para interagir numa posição trans com os orbitais de fronteira ( $\pi$ e $\pi^{*}$ ) do norborneno ( $\pi \rightarrow$ LUMO; HOMO $\rightarrow \pi^{*}$ e HOMO- $1 \rightarrow \pi^{*}$ ), o que pode ser visto na Figura $4 \mathrm{~S}$, material suplementar.

\section{Propagação}

As energias das espécies presentes durante a trimerização do norborneno (Tabela 3) mostram que a cada adição de uma nova molécula há uma estabilização do sistema. Isto condiz com resultados de trabalhos anteriores, ${ }^{20}$ que apontam um favorecimento nas taxas de reação do processo à medida que o radical $\mathrm{R}$ é aumentado (Figuras 2 e 1S).

Tabela 3. Energias relativas calculadas (B3LYP/lanl2dz) para as espécies envolvidas na trimerização do norborneno

\begin{tabular}{cccc}
\hline Estrutura & E/kcal mol ${ }^{-1}$ & Estrutura & E/kcal mol ${ }^{-1}$ \\
\hline catalisador & 0,0 & $\mathrm{f}_{2}$ & $-16,1$ \\
$\mathrm{a}$ & 23,2 & $\mathrm{c}_{3}$ & $-19,8$ \\
$\mathrm{~b}_{1}$ & $-1,4$ & $\mathrm{~d}_{3}$ & $-26,4$ \\
$\mathrm{c}_{1}$ & 16,3 & $\mathrm{e}_{3}$ & $-42,1$ \\
$\mathrm{~d}_{1}$ & 24,6 & $\mathrm{f}_{3}$ & $-33,1$ \\
$\mathrm{e}_{1}$ & $-9,9$ & $\mathrm{c}_{4}$ & $-39,6$ \\
$\mathrm{f}_{1}$ & $-0,1$ & $\mathrm{~d}_{4}$ & $-35,1$ \\
$\mathrm{c}_{2}$ & $-2,3$ & $\mathrm{e}_{4}$ & $-41,4$ \\
$\mathrm{~d}_{2}$ & 61,5 & $\mathrm{f}_{4}$ & $-26,2$ \\
$\mathrm{e}_{2}$ & $-25,2$ & trímero & $-49,4$ \\
\hline
\end{tabular}

A variação de energia observada na adição do primeiro norborneno pelo mecanismo dissociativo é de $-6,9 \mathrm{kcal} \mathrm{mol}^{-1}$. Na entrada do segundo norborneno, o valor observado é de $-2,1 \mathrm{kcal} \mathrm{mol}^{-1}$. Foram verificadas duas possíveis geometrias de entrada da terceira molécula de norborneno ( $c_{3} \mathrm{I}$ e $c_{3}$ II $)$ e do grupo eteno $\left(c_{4} \mathrm{I}\right.$ e $c_{4}$ II $)$, os valores de energia de estabilização observados são: $\mathrm{E}\left(c_{3} \mathrm{I}\right)=-3,7 ; \mathrm{E}\left(c_{3} \mathrm{II}\right)=-2,0$; $\mathrm{E}\left(c_{4} \mathrm{I}\right)=-6,5$ e $\mathrm{E}\left(c_{4} \mathrm{I}\right)=-2,2 \mathrm{kcal} \mathrm{mol}^{-1}$.

As adições sucessivas de olefinas ao catalisador modelo permitem um mecanismo exotérmico com barreiras energéticas pequenas.
Formação do estado de transição metalociclo

A segunda etapa da reação é o fechamento de um anel com o complexo $\pi\left(c_{x}\right)$ para formar um estado de transição metalocíclico $\left(d_{x}^{*}\right)$. A energética desta etapa pode ser vista na Figura 5.

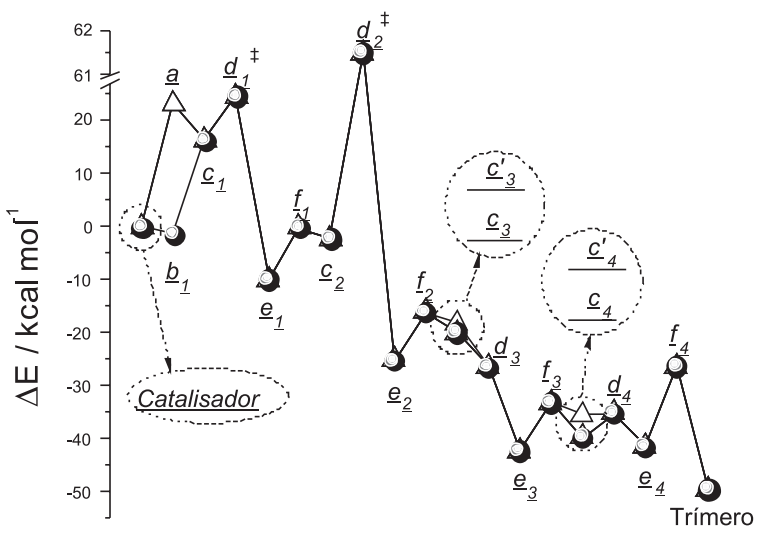

Figura 5. Perfil de energia completo para a trimerização do norborneno

Este passo envolve um giro da ligação $\pi$ da olefina, em relação à ligação $\mathrm{P}=\mathrm{C}$, que sofre uma rotação de $20,9^{\circ}$, abertura do ângulo $\mathrm{Cl}-\mathrm{Ru}-\mathrm{Cl}$ em torno de $22^{\circ}$ (em x $=1$, primeira olefina) e formação de uma nova ligação C-C. Um estado de transição é uma espécie molecular que é representada pelo topo de uma curva de energia potencial em uma coordenada reacional simples de uma dimensão. A energia desta espécie é necessária para a determinação da energia de ativação e, por sua vez, da velocidade da reação. A geometria de um estado de transição é um importante elemento informativo para a descrição do mecanismo de uma reação. Fomine ${ }^{20} \mathrm{em}$ um trabalho anterior utilizando B3LYP/lacvp** não conseguiu encontrar as estruturas de transição para propagação da cadeia na metátese ROMP do norborneno. Para verificar quais estruturas eram mínimos e quais se situavam em pontos de sela, foram feitos cálculos de frequências para todas as estruturas.

O complexo metalocíclico $d_{7}^{\ddagger}$ apresentou uma frequência imaginária com um valor de $189,8 \mathrm{i} \mathrm{cm}^{-1}$, e uma energia de ativação, $\mathrm{E} a_{l}$, igual a $8,3 \mathrm{kcal} \mathrm{mol}^{-1}$. Suresh ${ }^{21}$ e Janse, ${ }^{22}$ utilizando cálculos DFT para metátese do eteno com um catalisador metilideno, obtiveram energias de ativação semelhantes. A presença de uma frequência imaginária não garante que o estado de transição encontrado é realmente o máximo em energia numa coordenada que vai dos reagentes aos produtos; deste modo, foi realizado um cálculo IRC que confirmou que a estrutura $d_{1}^{\ddagger}$ era um estado de transição adequado.

A coordenação de mais olefinas (estruturas $c_{2}, c_{3}$, e $c_{4} /$ Figura 1S) se deu, em todos os casos, em uma configuração cis em relação à fosfina. Cálculos de densidade eletrônica total (Figura 5S, material suplementar) mostram que há um impedimento estérico provocado pelo volume molecular. Deste modo, uma adição trans em relação à fosfina não é possível nestes casos, apesar de ser eletronicamente favorável (como verificado em $c_{1}$ ). Outro impedimento verificado é a formação de uma ligação intramolecular entre um átomo de hidrogênio e o metal no sítio de coordenação trans, após a quebra da interação $\pi$ nas estruturas $e_{x}$. $\mathrm{O}$ arranjo estrutural não permite a orientação trans.

Encontrou-se um estado de transição $\left(d_{2}^{*}\right)$, com frequência imaginária em $237,3 \mathrm{i} \mathrm{cm}^{-1}$. Este manteve a orientação cis em relação à fosfina. A energia de ativação da etapa $c_{2} \rightarrow d_{2}$ foi de $63,8 \mathrm{kcal} \mathrm{mol}^{-1}$. Os cálculos IRC mostram que $d_{2}^{\ddagger}$ é um estado de transição entre $c_{2}$ e $e_{2}$. $\mathrm{O}$ valor de energia maior deve-se à forma de entrada do norborneno nesta etapa; na geometria obtida para estrutura $c_{2}$ a ligação $\pi$ do norborneno está ortogonal em relação ao grupo $\mathrm{Ru}=\mathrm{C}$, sendo necessário um giro de $90^{\circ}$ que dificulta o processo. 
Os cálculos de geometrias para coordenação nos complexos $\pi$ $c_{3}$ e $c_{4}$ (norborneno-catalisador e eteno-catalisador, respectivamente) indicam duas possíveis estruturas para a entrada da olefina, em cada caso (estruturas $c_{3}$ e $d_{3}, c_{4}$ e $d_{4}$ ). As estruturas $c_{3}$ e $d_{3}$ diferem quanto ao diedro formado entre os átomos $\mathrm{P}-\mathrm{Ru}-\mathrm{C}=\mathrm{C}$, os valores encontrados foram 12,4 e $47,0^{\circ}$, respectivamente. Os cálculos de frequências para estrutura $e_{3}$ exibem frequência imaginária em $3,2 \mathrm{i} \mathrm{cm}$, o que não corresponde a um estado de transição adequado neste mecanismo. As estruturas $f_{3} e d_{4}$ apesar de serem máximos num perfil energético que vai de $c_{3}$ a $e_{4}$ não foram caracterizados como estado de transição.

Nas possíveis geometrias apresentadas pelo complexo $\pi$, envolvido na finalização da reação, o eteno apresenta uma orientação $c i s$ em relação à fosfina, a diferença entre as estruturas está no grupo metal-carbeno que se apresenta com orientações cis e trans em relação à fosfina, estruturas $c_{4}$ e $d_{4}$, respectivamente. A variação total de energia nesta última etapa $\left(c_{4} \rightarrow d_{4}\right)$ foi de $1,8 \mathrm{kcal} \mathrm{mol}^{-1}$.

Tanto o norborneno quanto o catalisador adotado são estruturas simétricas, mas uma análise detalhada permite inferir que a cada adição de olefina ocorre uma inversão da configuração inicial, como pode ser visto na Figura 2, entre as estruturas $b_{x}$ e $f_{x}$.

\section{CONCLUSÕES}

Usando cálculos DFT foram verificados dois caminhos para dissociação da fosfina na metátese ROMP do norborneno envolvendo o modelo simplificado $\mathrm{Cl}_{2}\left(\mathrm{PH}_{3}\right)_{2} \mathrm{Ru}=\mathrm{CH}_{2}$. A geometria obtida para o catalisador modelo é semelhante a catalisadores de Grubbs metilideno reais e os perfis de energia calculados para dissociação de um catalisador metilideno real (com $\mathrm{L}=\mathrm{PCy}_{3}$ ) são semelhantes aos da estrutura modelo, com valores de energia menores. A dissociação de uma das fosfinas seguida da formação de uma ligação $\pi$ do norborneno em uma orientação trans em relação à outra fosfina é favorecida por diversos fatores. Tal coordenação é precedida por um mecanismo associativo seguido de uma dissociação fosfínica. A entrada de outros grupos olefínicos se dá com orientação cis em relação à fosfina presente, fato que se deve ao impedimento estérico presente na estrutura e a uma ligação intermolecular H-Metal no sítio de ligação trans, os efeitos estéricos não foram significativos na etapa inicial do processo. Fatores eletrônicos estão diretamente relacionados às etapas iniciais de interação catalisador-olefina e às estruturas geométricas. Um aumento da cadeia do radical olefínico presente na estrutura favorece a reação à medida que estabiliza o sistema. $\mathrm{O}$ mecanismo modelo é exotérmico e favorável energeticamente. Foram encontrados e confirmados dois estados de transição $\left(d_{1}^{\ddagger}\right.$ e $\left.d_{2}^{*}\right)$. Os cálculos mostram duas possíveis formas de interação catalisador-olefina para entrada da terceira molécula de norborneno e do grupo eteno.

\section{MATERIAL SUPLEMENTAR}

O mecanismo, em detalhes, proposto para reação, as figuras de orbitais, potencial eletrostático e densidade eletrônica, estão disponíveis em http://quimicanova.sbq.org.br, na forma de arquivo PDF, com acesso livre.

\section{AGRADECIMENTOS}

Ao CNPq pelo auxílio financeiro concedido para disponibilidade do programa necessário aos cálculos (Processo: 470341/2004-1).

\section{REFERÊNCIAS}

1. Frederico, D.; Brocksom, U.; Brocksom, T. J.; Quim. Nova 2005, 28, 692; Matos, J. M. E.; Batista, N. C.; Carvalho, R. M.; Santana, S. A. A.; Puzzi, P. N.; Sanches, M.; Lima-Neto, B. S.; Quim. Nova 2007, 30, 431.
2. Fomine, S.; Vargas, S. M.; Tlenkopatchev, M. A.; Organometallics 2002, $22,93$.

3. Riegler, S.; Demel, S.; Trimmel, G.; Slugovc, C.; Stelzer, F.; J. Mol. Catal. A: Chem. 2006, 257, 53.

4. Nguyen, S. T.; Johnson, L. K.; Grubbs, R. H.; Ziller, J. W.; J. Am. Chem. Soc. 1992, 114, 3974.

5. Schwab, P.; France, M. B.; Ziller, J. W.; Grubbs, R. H.; Angew. Chem., Int. Ed. 1995, 34, 2039.

6. Damrauer, N. H.; Cerullo, G.; Yeh, A.; Boussie, T. R.; Shank, C. V.; McCusker, J. K.; Science 1997, 275, 54; Henry, W.; Coates, C. G.; Brady, C.; Ronayne, K. L.; Matousek, P.; Towrie, M.; Botchway, S. W.; Parker, A. W.; Vos, J. G.; Browne, W. R.; McGarvey, J. J.; J. Phys. Chem. A 2008, 112, 4537; Mathies, R. A.; Lin, S. W.; Ames, J. B.; Pollard, W. T.; Annual Review of Biophysics and Biophysical Chemistry 1991, 20, 491.

7. Sanford, M. S.; Love, J. A.; Grubbs, R. H.; J. Am. Chem. Soc. 2001, 123, 6543.

8. Frisch, M. J.; Trucks, G. W.; Schlegel, H. B.; Scuseria, G. E.; Robb, M. A.; Cheeseman, J. R.; Montgomery Jr, J. A.; Vreven, T.; Kudin, K. N.; Burant, J. C.; Millam, J. M.; Iyengar, S. S.; Tomasi, J.; Barone, V.; Mennucci, B.; Cossi, M.; Scalmani, G.; Rega, N.; Petersson, G. A.; Nakatsuji, H.; Hada, M.; Ehara, M.; Toyota, K.; Fukuda, R.; Hasegawa, J.; Ishida, M.; Nakajima, T.; Honda, Y.; Kitao, O.; Nakai, H.; Klene, M.; Li, X.; Knox, J. E.; Hratchian, H. P.; Cross, J. B.; Adamo, C.; Jaramillo, J.; Gomperts, R.; Stratmann, R. E.; Yazyev, O.; Austin, A. J.; Cammi, R.; Pomelli, C.; Ochterski, J. W.; Ayala, P. Y.; Morokuma, K.; Voth, G. A.; Salvador, P.; Dannenberg, J. J.; Zakrzewski, V. G.; Dapprich, S.; Daniels, A. D.; Strain, M. C.; Farkas, O.; Malick, D. K.; Rabuck, A. D.; Raghavachari, K.; Foresman, J. B.; Ortiz, J. V.; Cui, Q.; Baboul, A. G.; Clifford, S.; Cioslowski, J.; Stefanov, B. B.; Liu, G.; Liashenko, A.; Piskorz, P.; Komaromi, I.; Martin, R. L.; Fox, D. J.; Keith, T.; AlLaham, M. A.; Peng, C. Y.; Nanayakkara, A.; Challacombe, M.; Gill, P. M. W.; Johnson, B.; Chen, W.; Wong, M. W.; Gonzalez, C.; Pople, J. A.; Gaussian 03, Revision B.02. 2003, Gaussian, Inc.: Pittsburgh PA.

9. Lee, C.; Yang, W.; Parr, R. G.; Phys. Rev. B 1988, 37, 785; Becke, A. D.; J. Chem. Phys. 1993, 98, 5648.

10. Filho, R. C. D. M.; Moura, E. M. d.; Souza, A. A. d.; Rocha, W. R.; J. Mol. Struct.: THEOCHEM. 2007, 816, 77.

11. Pastre, J. C.; Correia, C. R. D.; Quim. Nova 2008, 31, 872.

12. Scott Bohle, D.; Clark, G. R.; Rickard, C. E. F.; Roper, W. R.; Wright, L. J.; J. Organomet. Chem. 1988, 358, 411.

13. Louie, J.; Grubbs, R. H.; Organometallics 2002, 21, 2153.

14. Nguyen, S. T.; Grubbs, R. H.; Ziller, J. W.; J. Am. Chem. Soc. 1993, 115, 9858 .

15. Adlhart, C.; Chen, P.; J. Am. Chem. Soc. 2004, 126, 3496; Rowley, C. N.; Foucault, H. M.; Woo, T. K.; Fogg, D. E.; Organometallics 2008, 27, 1661; Cavallo, L.; J. Am. Chem. Soc. 2002, 124, 8965; Rensburg, W. J. v.; Steynberg, P. J.; Kirk, M. M.; Meyer, W. H.; Forman, G. S.; J. Organomet. Chem. 2006, 691, 5312; Adlhart, C.; Hinderling, C.; Baumann, H.; Chen, P.; J. Am. Chem. Soc. 2000, 122, 8204.

16. Sergei, F. V.; Michael, B.; Walter, T.; Chem.--Eur. J. 2002, 8, 3962.

17. Bernd, F. S.; Adv. Synth. Catal. 2007, 349, 204; Thomas, W.; Florian, J. K.; Wolfgang, H.; Dieter, G.; Wolfgang, A. H.; Angew. Chem., Int. Ed. 1999, 38, 2416; Matos, J. M. E.; Lima-Neto, B. S.; J. Mol. Catal. A: Chem. 2004, 222, 81 .

18. Silva Sá, J. L.; Lima-Neto, B. S.; J. Mol. Catal. A: Chem. 2009, 304, 187.

19. Dias, E. L.; Nguyen, S. T.; Grubbs, R. H.; J. Am. Chem. Soc. 1997, 119, 3887 .

20. Fomine, S.; Ortega, J. V.; Tlenkopatchev, M. A.; J. Mol. Catal. A: Chem. $\mathbf{2 0 0 5}, 236,156$

21. Suresh, C. H.; Koga, N.; Organometallics 2003, 23, 76.

22. Rensburg, W. J. v.; Steynberg, P. J.; Meyer, W. H.; Kirk, M. M.; Forman, G. S.; J. Am. Chem. Soc. 2004, 126, 14332. 


\section{INVESTIGAÇÃO DO MECANISMO DE CATÁLISE ROMP DO NORBORNENO UTILIZANDO MÉTODOS DE} FUNCIONAL DE DENSIDADE

\section{Carlos Pereira da Silva*}

Instituto Federal de Educação, Ciência e Tecnologia do Piauí, Campus Prof. Marcílio Rangel Farias, 64018-000 Teresina - PI, Brasil Francisco das Chagas Alves Lima

Coordenação de Química, Universidade Estadual do Piauí, 64002-150 Teresina - PI, Brasil

Régis Casimiro Leal e José Machado Moita Neto

Departamento de Química, Centro de Ciências da Natureza, Universidade Federal do Piauí, 64049-550 Teresina - PI, Brasil
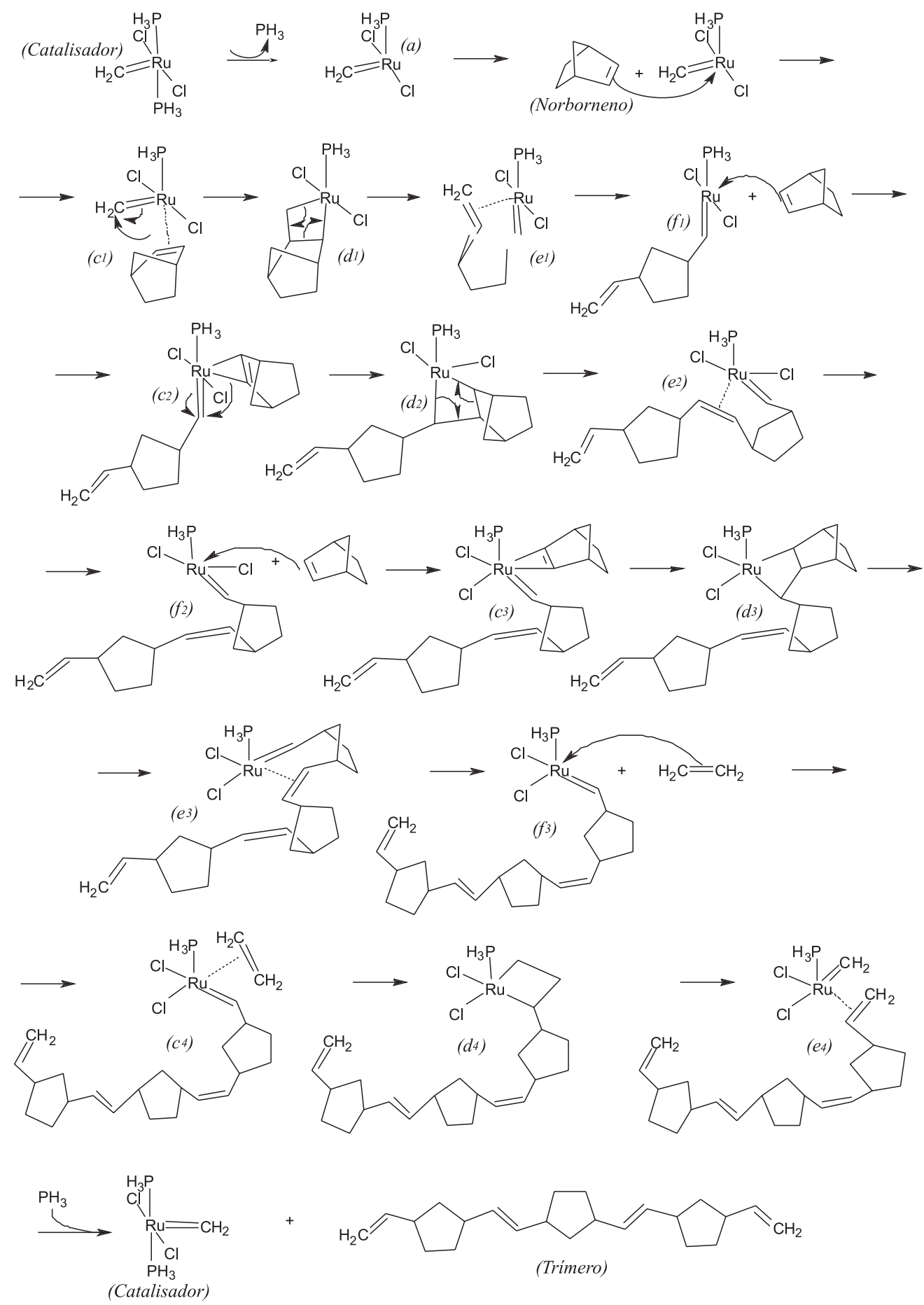

Figura 1S. Mecanismo proposto para trimerização do norborneno 

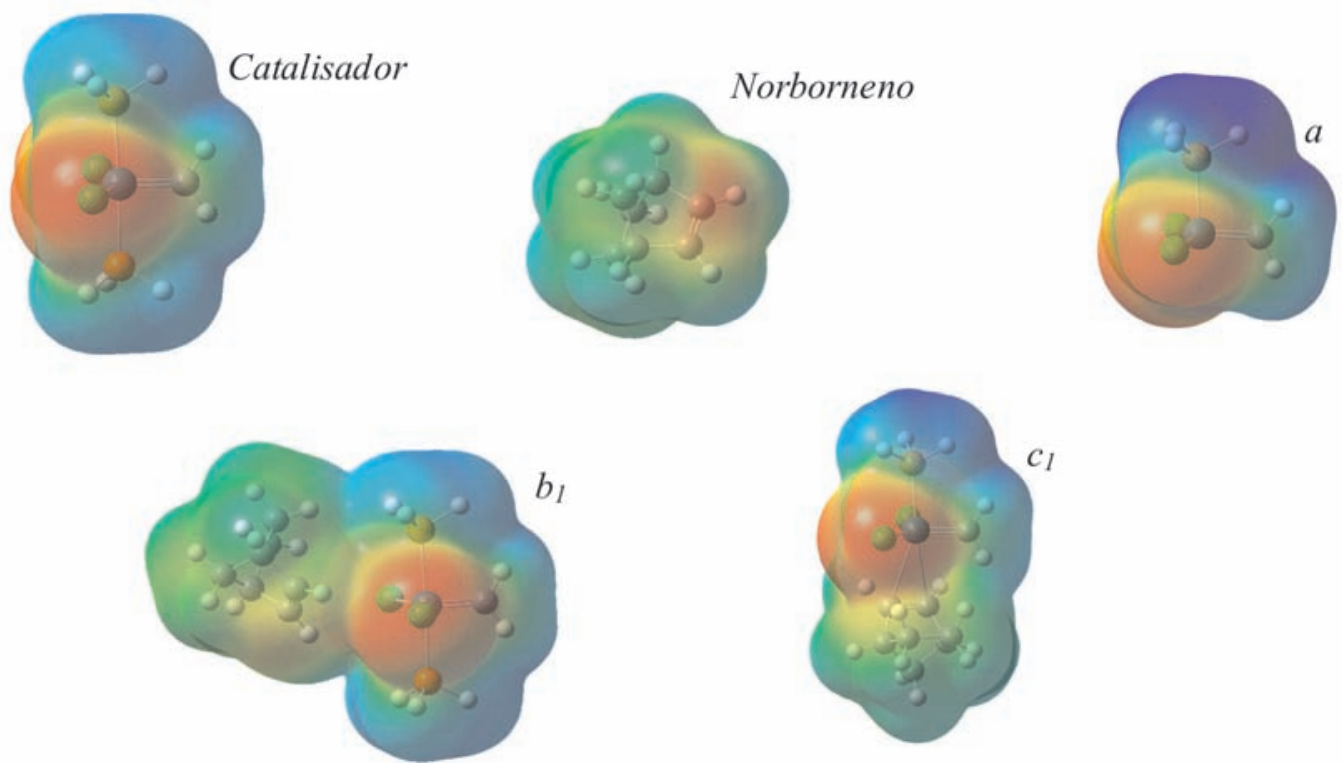

Figura 2S. SPE's calculadas (B3LYP/lanl2dz) para espécies envolvidas na dissociação da fosfina (-+)
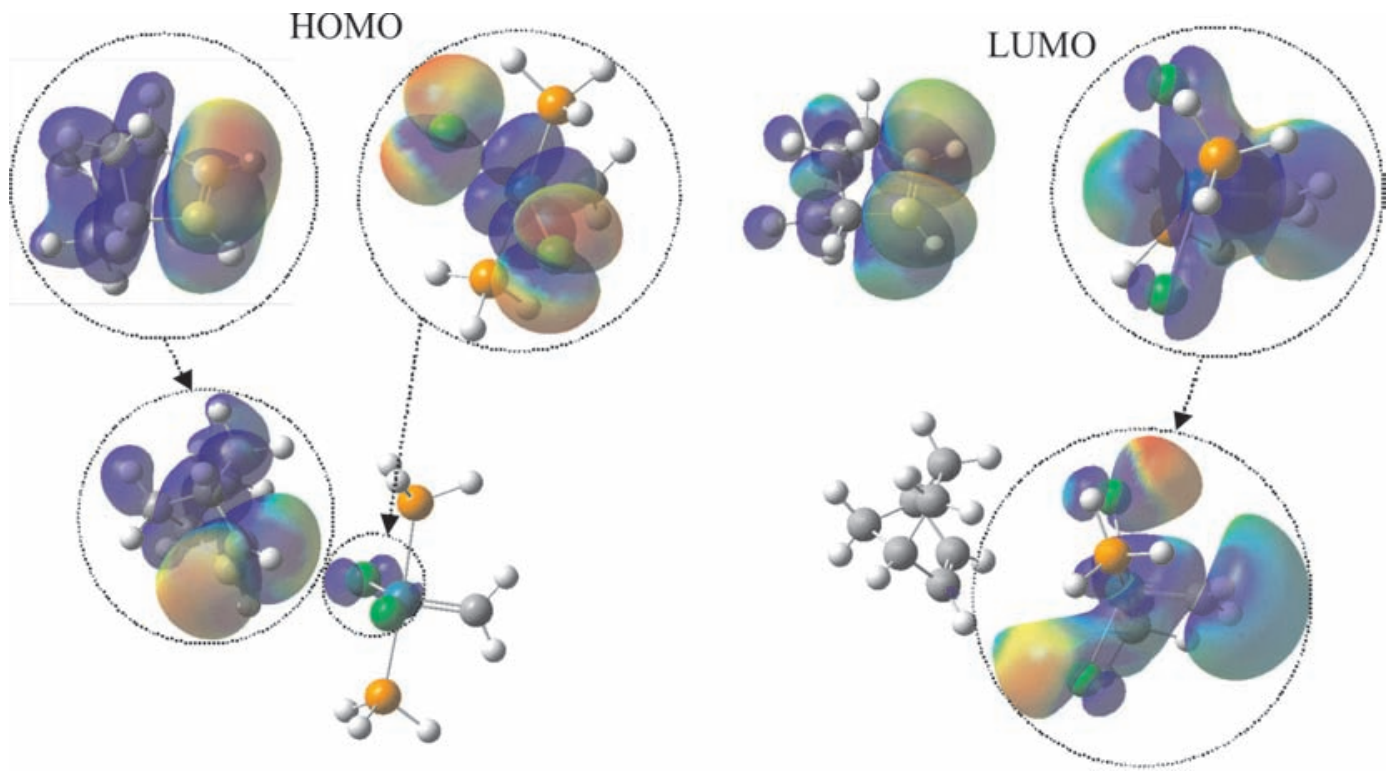

Figura 3S. Formação dos orbitais de fronteira HOMO e LUMO da estrutura $b_{1}($ B3LYP/LANL2DZ) (-+) 

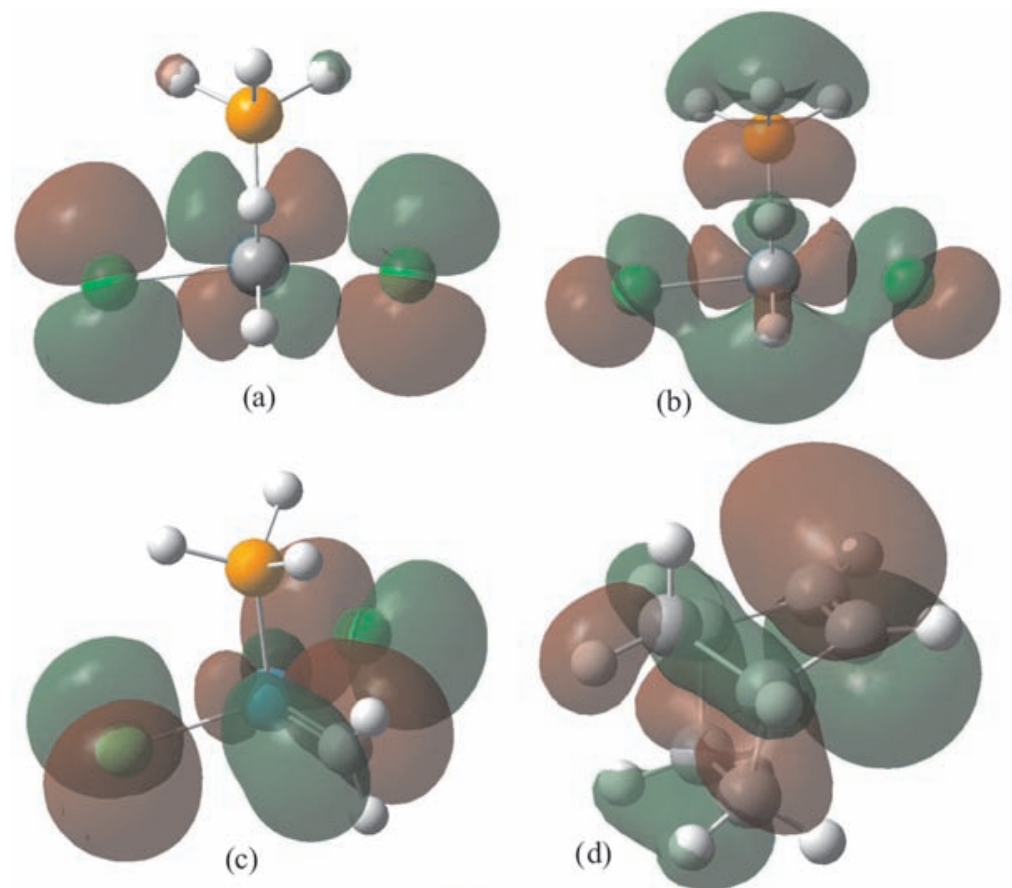

(c)

(e)

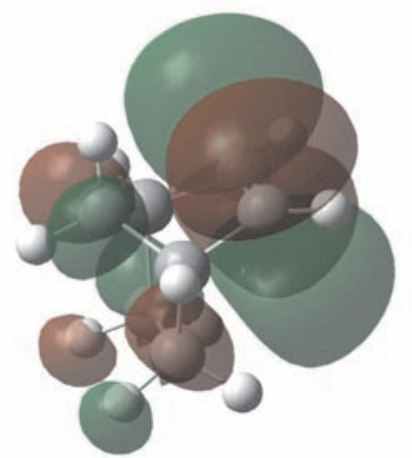

Figura 4S. Orbitais de fronteira das estruturas " a” - LUMO (a), HOMO (b), e HOMO-1 (c); e norborneno - $\pi(d)$ e $\pi *(e)(B 3 L Y P / L A N L 2 D Z)$

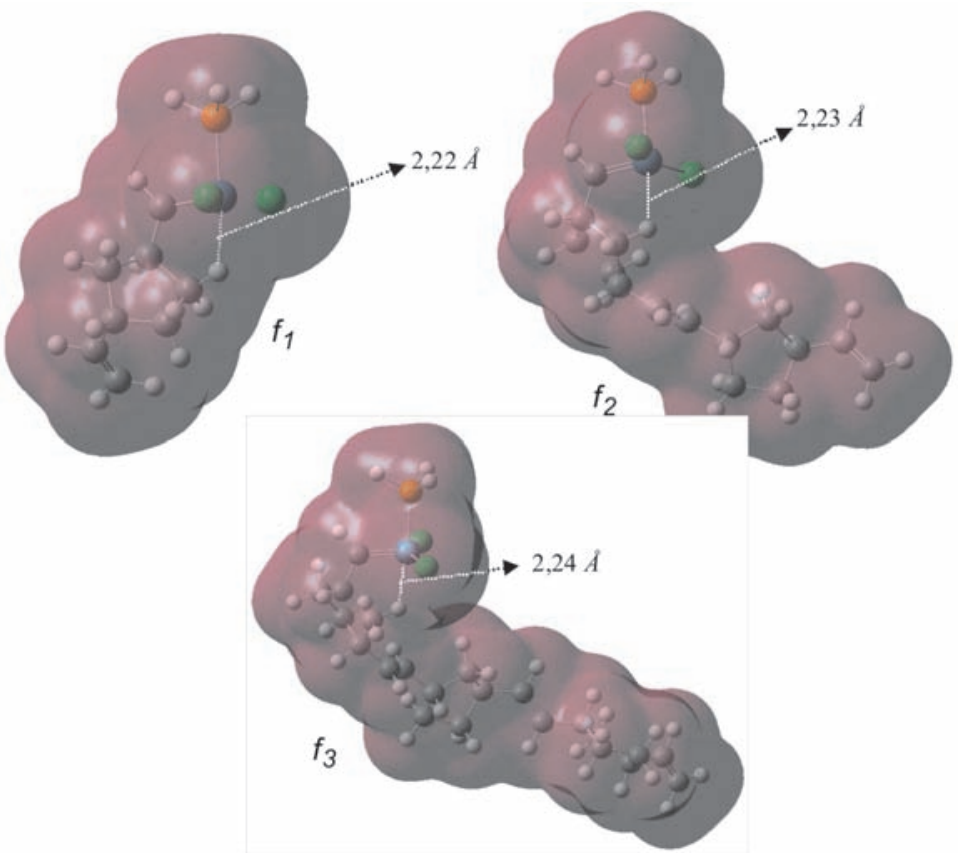

Figura 5S. Densidades eletrônicas totais (B3LYP/lanl2dz) calculadas para as estruturas $f_{1}, f_{2}$ e $f_{3}$ 